\title{
Honokiol exhibits enhanced antitumor effects with chloroquine by inducing cell death and inhibiting autophagy in human non-small cell lung cancer cells
}

\author{
XIAOQIN LV*, FANG LIU*, YUE SHANG and SHU-ZHEN CHEN \\ Institute of Medicinal Biotechnology, Chinese Academy of Medical Sciences \\ and Peking Union Medical College, Chongwen, Beijing 100050, P.R. China
}

Received April 16, 2015; Accepted June 3, 2015

DOI: 10.3892/or.2015.4091

\begin{abstract}
Honokiol (HNK), a potential antitumor compound, has been widely studied in recent years. It induces apoptosis and affects autophagy in cancer cells, yet the mechanism of its antitumor efficacy remains obscure. Chloroquine (CQ), an autophagy inhibitor, is often applied to sensitize antitumor drugs in clinical trials. Here, we investigated the antitumor effect of HNK or CQ alone or in combination in non-small cell lung cancer (NSCLC) cells. Using an experimental approach, 3-(4,5-dimethylthiazol-2-yl)-2,5-diphenyltetrazolium bromide (MTT) or sulforhodamine B (SRB) was used to determine the cytotoxicity of the agents. The expression levels of proteins were detected by western blotting. Apoptosis was examined via Annexin V-FITC and PI staining. H460 cell xenografts in nude mice were used to study the effects of HNK and/or CQ in vivo. Transfection with siRNA was applied to knock down cathepsin D. The results demonstrated the enhanced effects of HNK combined with CQ on the inhibition of proliferation, induction of apoptosis in vitro and the reduction in growth in vivo. It was confirmed that HNK and/or CQ triggered apoptosis via a caspase-dependent manner. Furthermore, HNK significantly increased the expression of p62 and LC3-II in the A549 and H460 cells and inhibited autophagy and induced
\end{abstract}

Correspondence to: Professor Shu-Zhen Chen, Institute of Medicinal Biotechnology, Chinese Academy of Medical Sciences and Peking Union Medical College, 1 Tiantan Xili, Chongwen, Beijing 100050, P.R. China

E-mail: bjcsz@imb.pumc.edu.cn

${ }^{*}$ Contributed equally

Abbreviations: HNK, honokiol; CQ, chloroquine; 3-MA, 3-methyladenine; MTT, 3-(4,5-dimethylthiazol-2-yl)-2,5-diphenyltetrazolium bromide; CDI, coefficient of drug interaction; SRB, sulforhodamine B; DMSO, dimethyl sulfoxide; zVAD.fmk, benzyloxycarbonyl-ValAla-Asp (OMe) fluoromethylketone; HCQ, hydroxychloroquine; PepA, pepstatin A

Key words: honokiol, chloroquine, antitumor effect, apoptosis, autophagy apoptosis in a cathepsin D-involved manner. In conclusion, an enhanced antitumor effect was demonstrated following treatment with HNK combined with CQ by inhibiting autophagy and inducing apoptosis via a caspase-dependent and cathepsin D-involved manner. This combination may be a novel and useful antitumor approach for chemotherapy in NSCLC.

\section{Introduction}

Honokiol (HNK) (Fig. 1A), a biphenolic compound isolated from the leaves, bark, and root of Magnolia officinalis is used in traditional Chinese and Japanese medicine (1). It was reported that HNK effectively reduces the growth of cancer in vitro and in vivo in cell and animal xenograft models (2). It also induces caspase-dependent apoptosis of cancer cells through downregulation of survivin, c-FLIP, Bcl-xL and upregulation of Bax and Bak (3-5). Recent studies have demonstrated that HNK increases the expression levels of the two hallmarks of autophagy, Beclin-1 and LC3-II, and induces autophagy in glioblastoma multiforme (GBM) DBTRG-05MG cells, yet the role of autophagy in the anti-GBM effects requires further research (6). In addition, HNK was found to induce autophagy in prostate cancer cells (7). However, another study indicated that w007B, a newly synthesized derivative of honokiol, exerted neuroprotective effects through inhibition of autophagy (8). As suggested above, the role of autophagy in the antitumor effects of HNK requires further research and combined treatment of HNK with an autophagy inhibitor or activator may result in enhanced antitumor effects.

Autophagy is a conserved, lysosomal-dependent membrane process to maintain cellular homeostasis under metabolic stress. During this process, autophagosomes engulf damaged proteins and defective organelles and then fuse with lysosomes for degradation (9). Finally, the unnecessary proteins are removed, the flawed organelles are eliminated and the cell growth is sustained during conditions of stress through autophagy. However, the role of autophagy in cancer is a double-edged sword (10). Nevertheless, the detailed molecular mechanisms of this dual action in tumors need to be elucidated. Chloroquine (CQ) diphosphate (Fig. 1B), an anti-malarial drug, reduces the proliferation, induces apoptosis and inhibits late stages of autophagy in cancer cells, and then 
exerts antitumor effects in vitro and in vivo. CQ and its analog hydroxychloroquine (HCQ) are the only autophagic inhibitors being used in clinical trials as a type of chemotherapy sensitizing agent (11). The inhibitors are evaluated by combining them with conventional chemotherapeutics or targeted agents for cancer therapy $(12,13)$. A combination of drugs is a major strategy for cancer chemotherapy. Thus, HNK combined with CQ may be a method by which to investigate the role of autophagy in the antitumor effect of HNK.

In the present study, we investigated the role of autophagy and apoptosis in the antitumor effects of HNK or CQ alone or their combination in non-small cell lung cancer (NSCLC) cells. The results showed that HNK combined with CQ exerted enhanced antitumor effects in vitro and in vivo through autophagy inhibition and apoptosis induction. They also demonstrated that HNK inhibited autophagy and induced apoptosis of NSCLC cells in a cathepsin D-involved and caspase-dependent manner.

\section{Materials and methods}

Antibodies and reagents. Rabbit anti-caspase- 3 and anti-PARP antibodies were purchased from Cell Signaling Technology (Beverly, MA, USA). The rabbit anti-LC3B antibody was obtained from Sigma (St. Louis, MO, USA). The mouse anti-cathepsin D antibody was obtained from Huada Biotechnology Co. (Beijing, China). The mouse anti- $\beta$-actin antibody was obtained from ZSGB-BIO (Beijing, China). CQ and 3-methyladenine (3-MA) were purchased from Sigma (Deisenhofen, Germany), prepared initially as a $20 \mathrm{mM}$ stock solution by dissolving in physiological saline (Minkang, China) and dissolved in RPMI-1640 medium to the desired concentration before the experiment, respectively. HNK was purchased from Shanghai Ziyi Reagent Factory (Shanghai, China) and rapamycin was purchased from Shanghai Qianchen Reagent Co. (Shanghai, China). The two agents were dissolved in dimethyl sulfoxide (DMSO) as a $20 \mathrm{mM}$ and $218 \mu \mathrm{M}$ stock, respectively. Before use in the experiments, each stock solution was diluted with RPMI-1640 medium to the desired final concentration. The caspase-3 inhibitor N-benzyloxycarbonylVal-Ala-Asp-fluoromethylketone (zVAD.fmk) was purchased from Beyotime (Jiangsu, China) and acridine orange was purchased from Sigma.

Cell culture. The human NSCLC cell lines A549 and H460 were used in the experiments and stored in our laboratory. All the cell lines were cultured in RPMI-1640 medium (Thermo Scientific) supplemented with $10 \%$ fetal calf serum (Gibco), $1 \%$ penicillin/streptomycin (North China Pharmaceutical, China) and maintained in a $5 \% \mathrm{CO}_{2}$ incubator at $37^{\circ} \mathrm{C}$.

Cell survival analysis. Cells were seeded in 96-well microtiter plates and exposed to different concentrations of the chemicals overnight for $48 \mathrm{~h}$. Then, the sulforhodamine B (SRB) or 3-(4,5-dimethylthiazol-2-yl)-2,5-diphenyltetrazolium bromide (MTT) assay was used to detect cell viability. The SRB assay is briefly described as follows. After the treatment period, the cells were fixed with cold $10 \%(\mathrm{w} / \mathrm{v})$ trichloroacetic acid and incubated for $60 \mathrm{~min}$ at $4^{\circ} \mathrm{C}$. After the supernatant was discarded, double distilled water was used to wash the plates
A
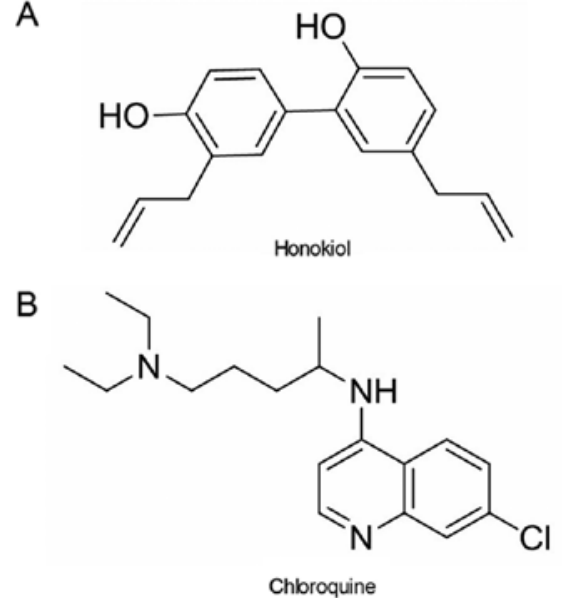

Figure 1. Chemical structure of (A) honokiol and (B) chloroquine.

five times and air dried. Then, SRB solution $[0.4 \%(\mathrm{w} / \mathrm{v})$ in $1 \%$ acetic acid)] was applied to stain the cells for $5 \mathrm{~min}$ at room temperature. After staining, the plates were washed with $1 \%$ acetic acid five times to remove the unbound dye and air dried. SRB was subsequently solubilized with $10 \mathrm{mM}$ Tris ( $\mathrm{pH} 10.5$ ), and the absorbance was read at $570 \mathrm{nM}$ on a microplate reader (Multiskan MK3; Thermo Scientific). The MTT assay was carried out as previously described (14).

Evaluation of the effects of the combined drugs. The coefficient of drug interaction (CDI) was applied to evaluate the effect of drug interaction. CDI was calculated as follows:

$$
\mathrm{CDI}=\mathrm{AB} /(\mathrm{A} \times \mathrm{B})
$$

Where $A B$ is the survival rate of the cells affected by the combined drugs. A or B is the survival rate of cells affected by each drug alone. When CDI is $<1$, there exists a synergistic effect of the two drugs (15).

Apoptosis analysis. Cells were plated into 6-well plates and were treated with different concentrations of the agents for $20 \mathrm{~h}$. Then, cell apoptosis was examined by the Annexin V-FITC/PI apoptosis detection kit (Biosea Biotechnology, Beijing, China) according to the manufacturer's instructions. Briefly, the cells were collected, fixed and stained with $10 \mu 1$ of Annexin V-FITC and $5 \mu \mathrm{l}$ of PI. Flow cytometric analysis was performed on $1 \times 10^{4}$ cells/sample by a FACS Coulter EPICS XL in less than $1 \mathrm{~h}$ and analyzed with a FACSCalibur using Summit software (both from Beckman Coulter, Inc., Fullerton, CA, USA). Caspase activation, an additional indicator of apoptosis, was also detected by western blotting as described below.

Western blot analysis. Cells were exposed to the chemicals for different times and then harvested. The cells were lysed and analyzed by western blotting as previously described (16).

In vivo efficacy studies. The protocols of animal experiments were, according to the rules of Good Laboratory Practice for Non-Clinical Laboratory Studies of Drugs issued by the National Scientific and Technologic Committee of P.R. China, approved by the Experimental Animal Center of the Institute of Medicinal Biotechnology. 
A

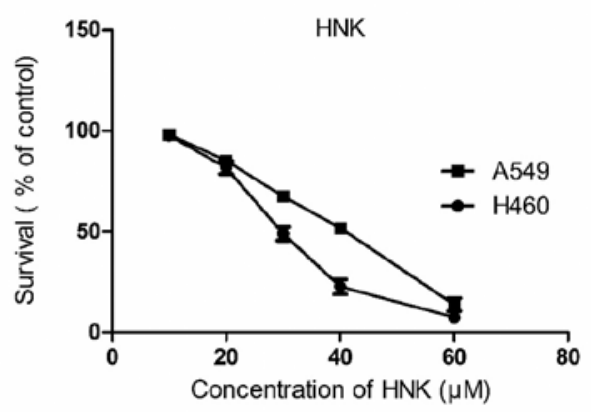

B

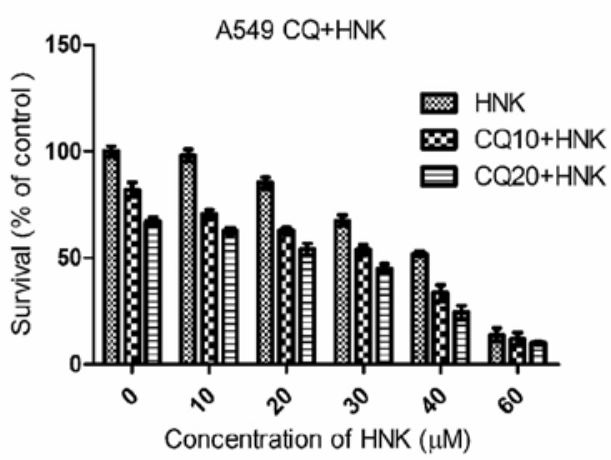

C

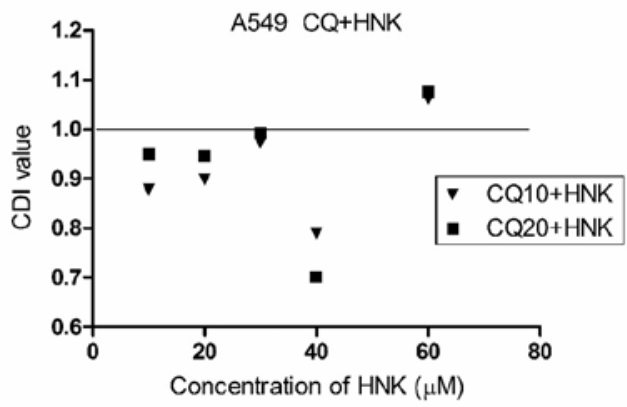

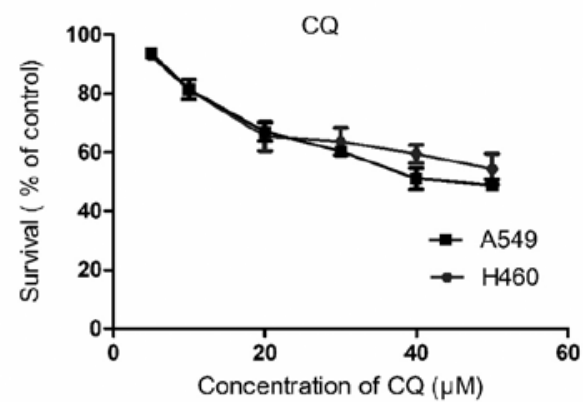
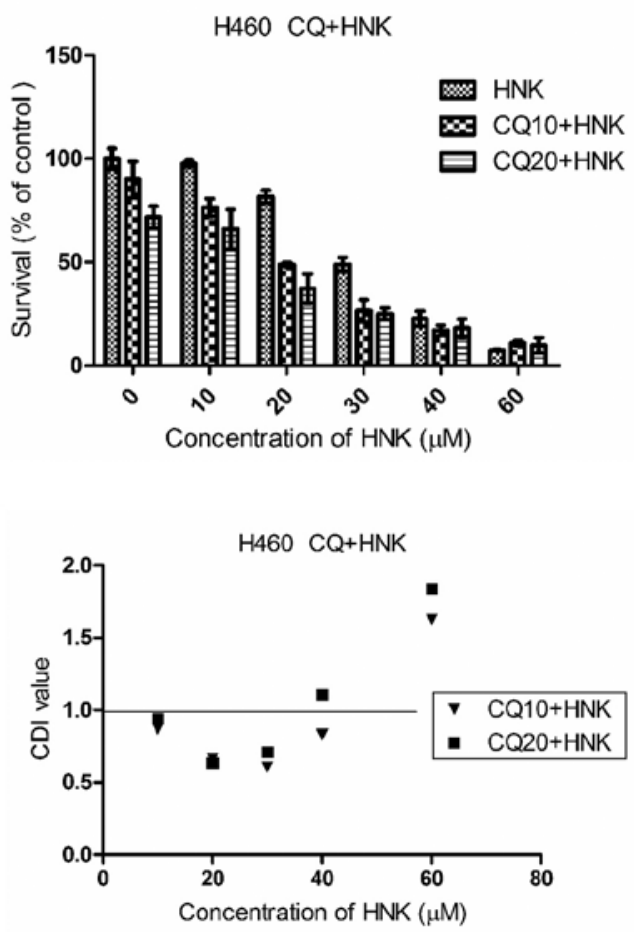

Figure 2. HNK and/or CQ inhibit the proliferation of NSCLC cells. (A) Proliferative activity of the H460 and A549 cells treated with HNK or CQ at the indicated concentrations for $48 \mathrm{~h}$ as assessed by the SRB assay. (B) The effect of CQ combined with HNK on the proliferative activity of the A549 and H460 cells as assessed by the SRB assay. Cells were treated with CQ (10 and $20 \mu \mathrm{M})$ and HNK (10, 20,30, 40 and $60 \mu \mathrm{M})$ for $48 \mathrm{~h}$. Data are expressed as the ratio to the control (untreated) cells. Values are expressed as mean \pm SD. (C) The CDI value is a quantitative measure of the degree of interaction between different drugs. CDI values between 1 and 0.7 indicate slight to moderate synergism and CDI values of 0.7-0.3, strong synergism. NSCLC, non-small cell lung cancer cells; HNK, honokiol; CQ, chloroquine; SRB, sulforhodamine B; CDI, coefficient of drug interaction.

We obtained 6- to 8-week-old female BALB/c nude mice (nu/nu) from Vital River Co. (Beijing, China) and housed them under pathogen-free conditions with laboratory chow and water. $\mathrm{H} 460$ cells $\left(5 \times 10^{6} /\right.$ mouse) were injected into the right armpits of the nude mice. After 3 weeks, tumors resulting from donor animals were aseptically dissected and cut into $2 \times 2 \times 2 \mathrm{~mm}^{3}$ pieces. The pieces were then transplanted into fresh mice using trocar needles. Prior to the tumors reaching $\sim 100 \mathrm{~mm}^{3}$, the mice were randomized into 5 groups (6/group) based on body weight and tumor volume. The mice were administered HNK (50 mg/kg/day, 5 times/week, i.p.) and CQ (100 mg/kg/day, 5 times/week, p.o.). Control nude mice were injected with saline or $20 \%$ intralipid (HNK dissolved in $20 \%$ intralipid) (17). The long and short diameters of the tumors ( $\mathrm{a}$ and $\mathrm{b}$, respectively) were measured using a caliper twice a week and the body weights were recorded at the same time. The tumor volume was calculated according to the formula, $\mathrm{V}=\mathrm{ab}^{2} / 2$. The animal experiment lasted for 21 days. At the end, the tumor tissues were used to assess the LC3-II expression with western blotting.

Acridine orange (AO) staining. The abundance of acidic lysosomes in the cells was evaluated with AO staining. After treatment with $\mathrm{HNK}$ for $24 \mathrm{~h}$, the cells were stained with acridine orange $(1 \mu \mathrm{g} / \mathrm{ml})$ for $15 \mathrm{~min}$ at $37^{\circ} \mathrm{C}$ in the dark and then washed with PBS. The images were captured using a fluorescence microscope (Vert. A1; Zeiss, Germany) using a 490-nm band-pass excitation filters and a 515-nm long-pass barrier filter. Based on the acidity, the lysosomes appeared as orange fluorescent cytoplasmic vesicles (18).

siRNA transfection. Experimentally siRNA targeting human cathepsin D (cathepsin D-siRNA) was purchased from Ruibo Biotechnology (Guangzhou, China), and the following siRNA 
A
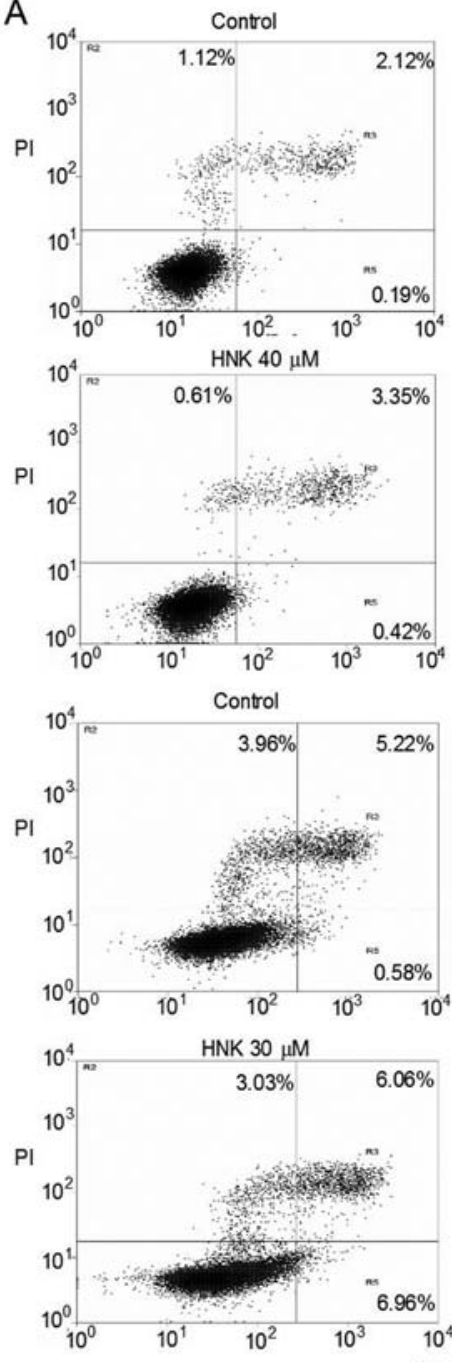
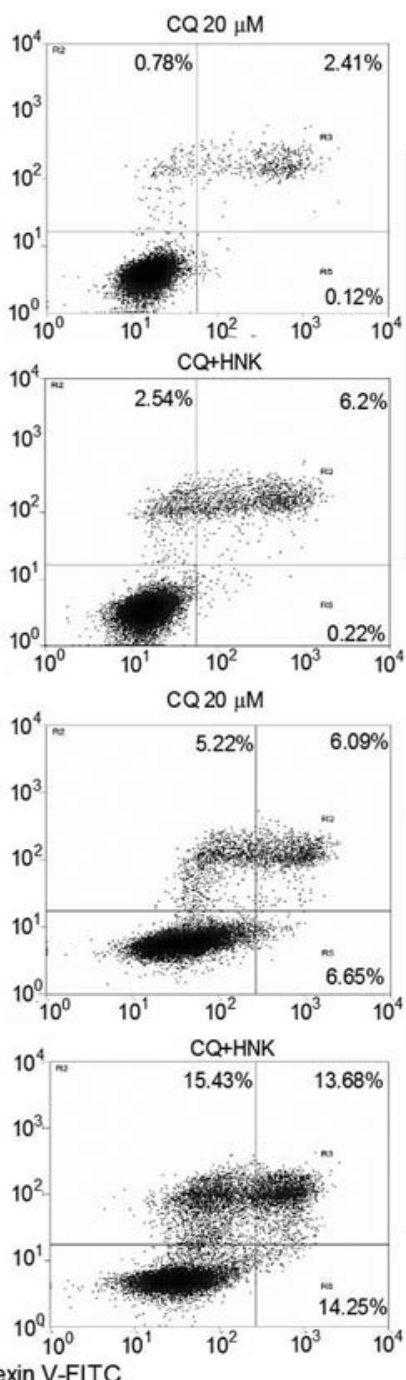

A549

$\mathrm{H} 460$

Figure 3. CQ or/and HNK induce the apoptosis of H460 and A549 cells in a caspase-dependent manner. Induction of apoptosis following treatment with HNK ( $40 \mu \mathrm{M}$ for the A549 cells and $30 \mu \mathrm{M}$ for the $\mathrm{H} 460$ cells) or CQ $(20 \mu \mathrm{M})$ alone or in combination for $24 \mathrm{~h}$ in the A549 and H460 cells. (A) Analysis of apoptosis was performed by Annexin V-FITC/PI apoptosis assay, as described in Materials and methods. HNK, honokiol; CQ, chloroquine.

sequence was CCUCGUUUGACAUCCACUAdTdT (19). The cells were transfected with siRNAs using the Ruibo FECT ${ }^{\mathrm{TM}}$ $\mathrm{CP}$ transfection kit according to the manufacturer's instructions. Briefly, the cells were plated in a 6-well plate for an overnight incubation, and then the medium was altered to antibiotic-free for transfection. After the transfected solution in an antibiotic-free condition was added to the plates, the cells were incubated at $37^{\circ} \mathrm{C}$ for $24 \mathrm{~h}$ and treated with $\mathrm{HNK}$ for another $24 \mathrm{~h}$. Finally, the efficiency of the RNAi was measured by western blotting.

Statistical analysis. All of the experiments were conducted independently at least three times. All the data are represented as mean $\pm \mathrm{SD}$.

\section{Results}

$H N K$ and/or CQ inhibit the proliferation of NSCLC cells. In order to investigate the antitumor effect of combining HNK with CQ, an SRB assay was used to validate the cytotoxicity of HNK or CQ alone or their combination. As shown in Fig. 2A,
HNK or CQ decreased the viability of the A549 and H460 cells in a dose-dependent manner at $48 \mathrm{~h}$.

Then the antitumor effect of the combination of HNK and $\mathrm{CQ}$ was investigated via the SRB assay. The cells were treated with CQ at the doses of 10 and $20 \mu \mathrm{M}$, and then doses of HNK were added at the same time. CQ sensitized the H460 and A549 cells to HNK (Fig. 2B) and the majority of the CDIs in the combination doses were $<1$ (Fig. 2C). Therefore, there was a synergistic effect between CQ and HNK.

HNK and/or CQ induce the cell death of H460 and A549 cells in a caspase-dependent manner. To determine the effect of the two drugs on the apoptosis of human NSCLC cells, both cell lines were treated with HNK (40 $\mu \mathrm{M}$ for the A549 and $30 \mu \mathrm{M}$ for the $\mathrm{H} 460$ cells) or CQ $(20 \mu \mathrm{M})$ alone or in combination for $24 \mathrm{~h}$. Annexin V-FITC and PI staining indicated early and late apoptotic and necrotic cells, respectively. The apoptotic cells were increased in the CQ+HNK group compared with the CQ or HNK group. The apoptotic percentages of the A549 cells were $2.53,3.77$ and $6.42 \%$ for $\mathrm{CQ}, \mathrm{HNK}$ and CQ+HNK, respectively, and the apoptotic 
B

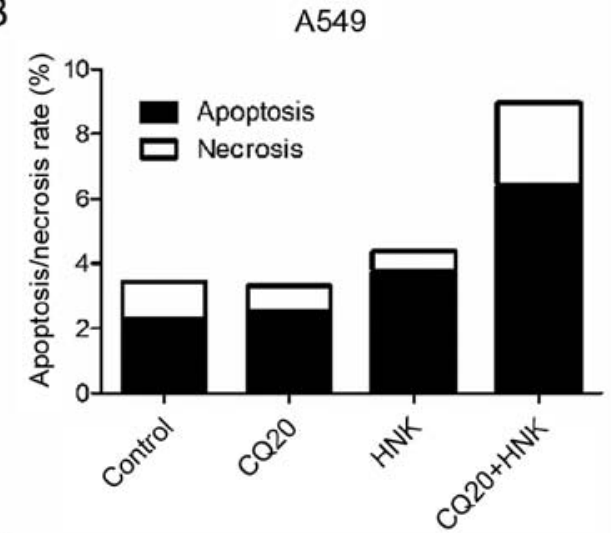

C

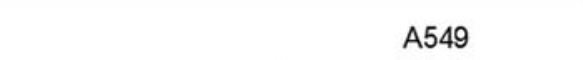

$\mathrm{H} 460$

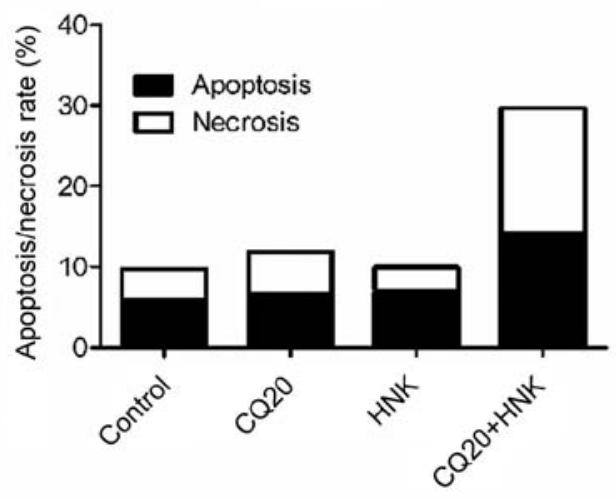

$\mathrm{H} 460$

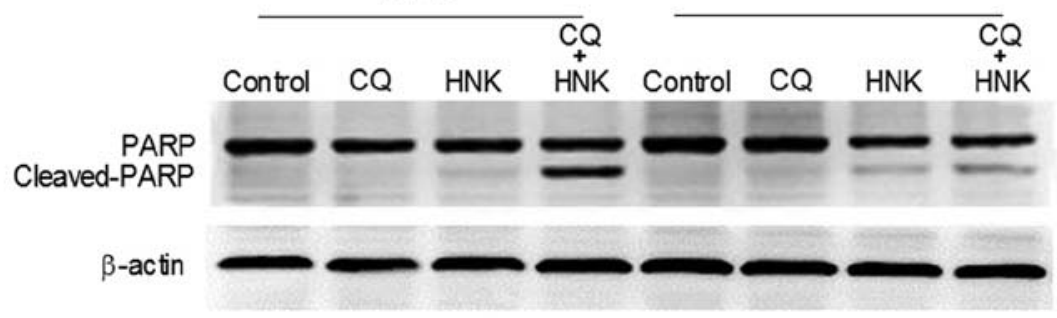

D

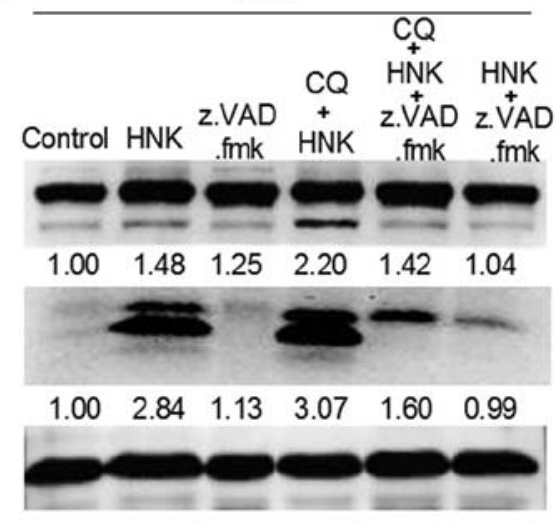

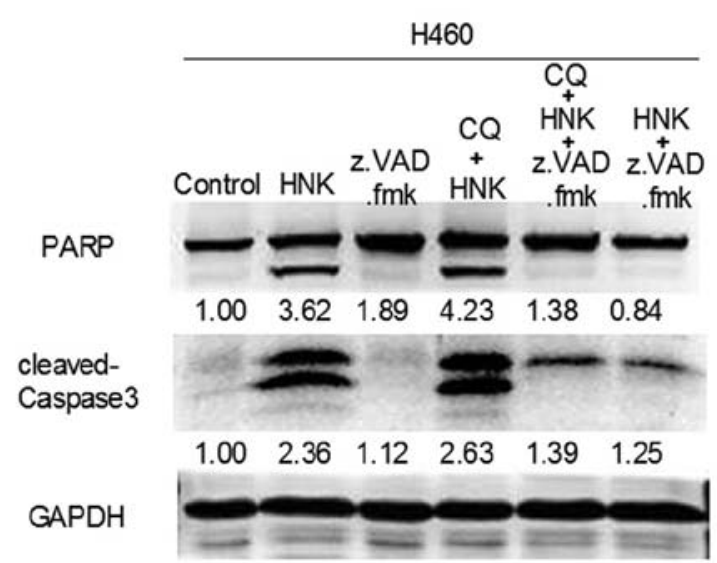

Figure 3. Continued. CQ and/or HNK induce the apoptosis of $\mathrm{H} 460$ and A549 cells in a caspase-dependent manner. Induction of apoptosis by treatment with HNK ( $40 \mu \mathrm{M}$ for the A549 cells and $30 \mu \mathrm{M}$ for the $\mathrm{H} 460$ cells) or CQ $(20 \mu \mathrm{M})$ alone or in combination for $24 \mathrm{~h}$ in the A549 and H460 cells. (B) The apoptosis and necrosis rate of the treated cells is shown. (C) The expression of proteins associated with apoptosis in the A549 and H460 cells was assessed by western blotting. The cells were exposed to CQ $(20 \mu \mathrm{M})$ and HNK (40 $\mu \mathrm{M}$ for the A549 cells and $30 \mu \mathrm{M}$ for the H460 cells) for $24 \mathrm{~h}$. (D) zVAD.fmk reduced the increased expression of cleaved-PARP and cleaved caspase-3 induced by HNK and/or CQ. zVAD.fmk $(100 \mu \mathrm{M})$ was used to pretreat the A549 and H460 cells for $1.5 \mathrm{~h}$, and then HNK and/or CQ or no agents were added for another $24 \mathrm{~h}$. The apoptosis-related proteins were assessed by western blotting. The number under each blot is the ratio of the optical density of the blot relative to that of the untreated group. HNK, honokiol; CQ, chloroquine.

percentages of the $\mathrm{H} 460$ cells were $6.65,6.96$ and $14.25 \%$, respectively (Fig. 3A and $\mathrm{B}$ ).

After the combination of CQ and HNK induced NSCLC cells to undergo apoptosis, western blotting was applied to investigate the levels of marker protein expression in the apoptotic pathway. The CQ+HNK group exhibited decreased PARP and increased cleavage after the two drugs were combined as compared to CQ or HNK alone (Fig. 3C).

Finally, zVAD.fmk, a broad-range caspase inhibitor, was used to examine whether the cell death induced by the combination of HNK and CQ was caspase-dependent. zVAD.fmk significantly decreased the HNK and CQ-triggered increased levels of cleaved caspase-3 and cleaved-PARP in the A549 and H460 cells (Fig. 3D).
$H N K$ and/or $C Q$ reduce the growth of the $H 460$ xenografts in $B A L B / c$ nude mice. The above results demonstrated an enhanced effect on the proliferation and apoptosis of NSCLC cells following the combination of HNK and CQ. Thus, we explored whether CQ can augment the antitumor effect of HNK in vivo. According to the method as previously described, administration of the drugs was started at day 7 after tumor blocks were implanted in the BALB/c nude mice. No incidence of death in the experimental groups occurred during the experiment. Fig. 4A shows that the average body weight in the groups did not significantly change and was stable. Thus, the doses of CQ and HNK were tolerated. The volume of the mouse tumors was reduced in the $\mathrm{CQ}, \mathrm{HNK}$ and $\mathrm{CQ}+\mathrm{HNK}$ groups as compared to the controls and the 
A
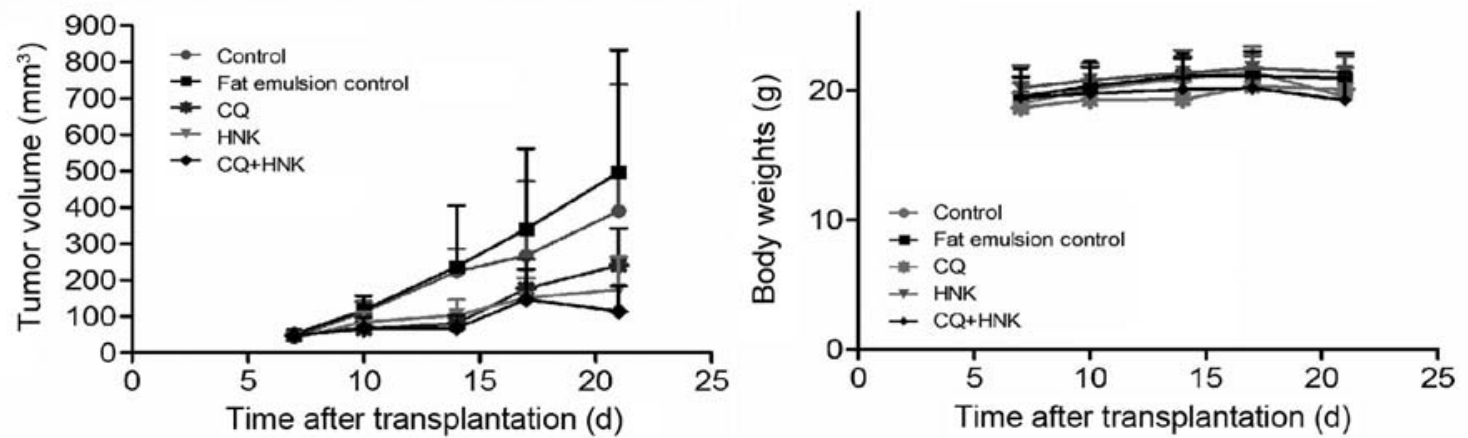

B

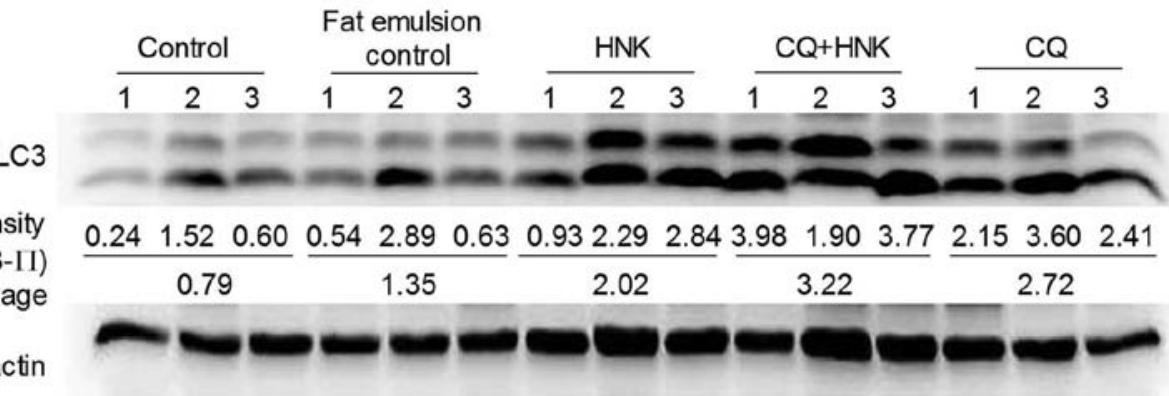

Figure 4. HNK and/or CQ reduce the growth of lung cancer $\mathrm{H} 460$ xenografts in BALB/C nude mice. Tumor-bearing mice were treated with $\mathrm{HNK}(50 \mathrm{mg} / \mathrm{kg} / \mathrm{day}$; i.p.) and $/$ or CQ (100 mg/kg, p.o.) after tumor transplantation as described in Materials and methods. (A) HNK and/or CQ decreased the growth of the H460 xenografts in nude mice, exhibited by changes in the tumor volume. Tumor volumes were measured twice a week. Values are expressed as mean \pm SD. Changes in body weight of nude mice bearing human lung cancer $\mathrm{H} 460$ xenografts are also shown. (B) Expression of LC3 in the tumor tissues was assessed by western blotting. The number under each band (LC3-II) is a ratio of the optical density of the band relative to that of its corresponding actin. HNK, honokiol; CQ, chloroquine.

percentage of tumor growth inhibition in the three groups on day 21 was 38.81, 64.92 and $76.91 \%$, respectively (Fig. 4A). Thus, the reduction in tumor volume in the CQ+HNK group was more significant than that in the CQ or HNK group.

It has been reported that HNK produces pharmacological functions via influencing autophagy, and CQ is used as an autophagic inhibitor during autophagy studies. Thus, we further investigated the LC3-II expression in tumor tissues from the $\mathrm{H} 460$ xenografts in $\mathrm{BALB} / \mathrm{c}$ nude mice. In the experiment, the tissues from three mice in each group were used to conduct western blotting. The results revealed that LC3-II expression was increased after CQ, HNK or CQ+HNK treatment. The average intensity of the LC3-II band in the $\mathrm{CQ}+\mathrm{HNK}$ group was greater than that in the CQ or HNK group (Fig. 4B).

HNK increases the expression of LC3-II and p62 in human NSCLC cells. From the above results, we found that HNK increased the expression of LC3-II similar to CQ. To further show the role of autophagy in the antitumor effects of HNK or coupled with CQ in NSCLC cells, the expression levels of p62 and LC3-II in the A549 and H460 cells were examined after HNK treatment. As shown in Fig. 5A, HNK at a concentration as low as $10 \mu \mathrm{M}$ was able to increase the p62 and LC3-II expression in the A549 cells, and it increased levels of the two proteins at the concentration of $20 \mu \mathrm{M}$ in the $\mathrm{H} 460$ cells. In addition, HNK increased the levels of LC3-II in the A549 and $\mathrm{H} 460$ cells, which occurred at $8 \mathrm{~h}$ and were maintained at least for $24 \mathrm{~h}$. The p62 expression level was significantly increased at $16 \mathrm{~h}$ and was maintained up to $24 \mathrm{~h}$ (Fig. 5B). From this, we found that prolonged treatment of HNK generated cell linedependent results on p62 and LC3-II regulation.

Autophagy is a lysosome-dependent membrane degradative process, thus the lysosome stability was examined by AO staining. During the experiment, the nucleus was stained green and acidic vesicles (lysosomes and autophagolysosomes) were present in red fluorescence. As shown in Fig. 5C, the red fluorescence intensity was increased in the cytoplasm of both cell lines as compared to that in the control group after HNK treatment for $24 \mathrm{~h}$.

HNK combined with autophagy inhibitors effects the proliferation and autophagy-related protein expression in human NSCLC cells. In order to investigate the role of autophagy in the antitumor effect of $\mathrm{HNK}$ or combined with CQ, various inhibitors were applied as tools to inhibit autophagy. In this experiment, CQ and 3-MA, as autophagy inhibitors at different stages, were used to examine the role of autophagy on the cytotoxicity induced by HNK. The lysosome-inhibitor CQ inhibited the fusion of lysosomes and autophagosomes, causing the accumulation of LC3. 3-Methyladenine (3-MA) inhibited the initiation of autophagy (20). At first, the cell viability was measured by MTT assay. HNK, CQ or 3-MA was able to suppress the proliferation of the A549 and H460 cells. The combination of HNK and CQ decreased the viability of both cell lines more potently than HNK or CQ alone, but there was no enhanced effect on the inhibition of proliferation by HNK or CQ combined with 3-MA, respectively (Fig. 6A).

Secondly, we conducted western blotting to detect the expression of p62 and LC3-II after HNK, CQ, 3-MA or the 

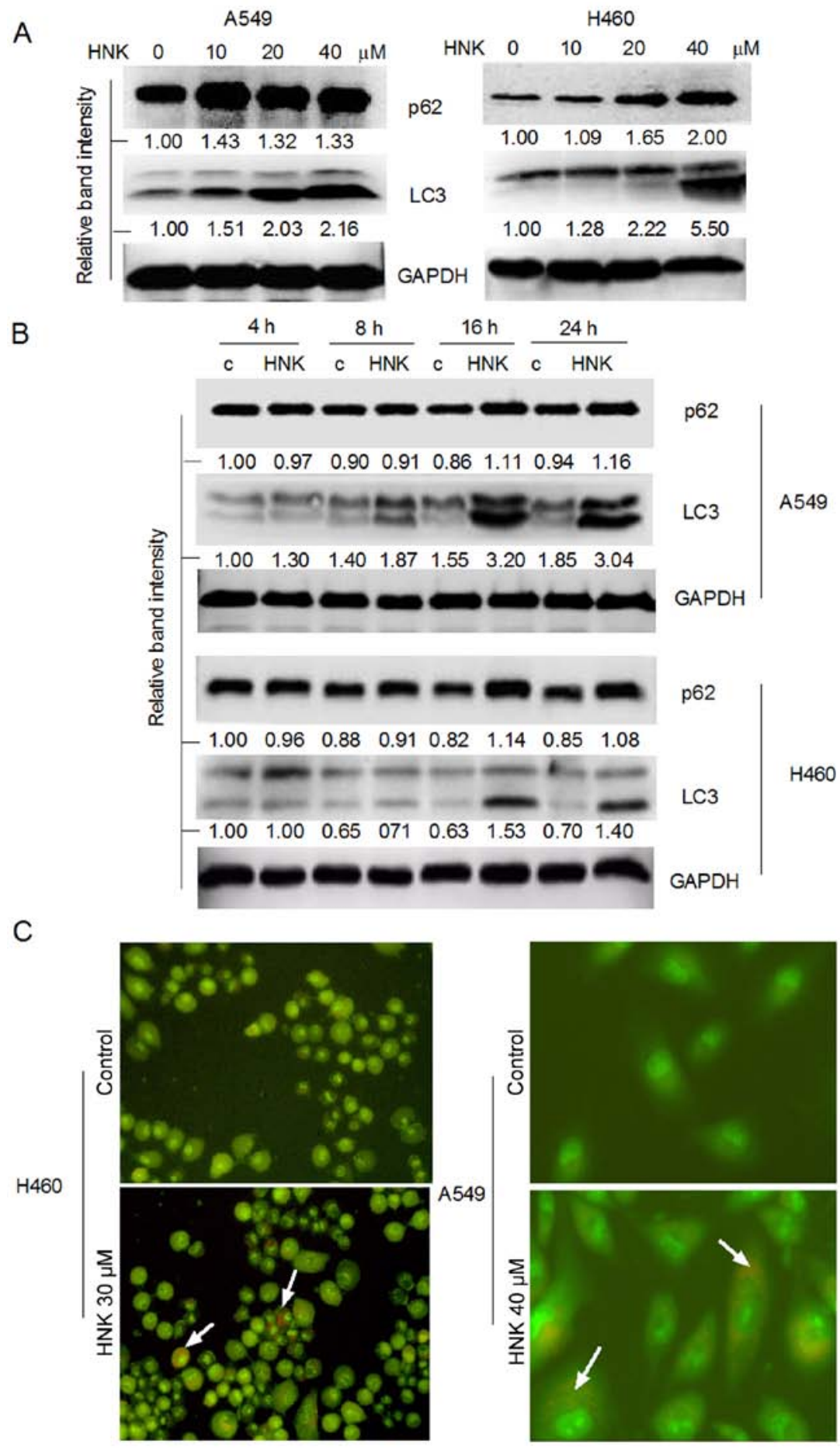

Figure 5. HNK blocks autophagy in the NSCLC cells. Various markers of autophagy were analyzed in the HNK-treated A549 and H460 cells. (A and B) The accumulation of p62 and LC3- I/II in the A549 and H460 cells treated with the indicated concentrations of HNK for $24 \mathrm{~h}$ (A) or with $40 \mu \mathrm{M}$ (A549 cells) and $30 \mu \mathrm{M}$ (H460 cells) HNK respectively for the indicated times (B). The expression of p62 and LC3-I/II was assessed by western blotting. The number under each band is the ratio of the optical density of the band relative to that of the untreated group (A) or the control at $4 \mathrm{~h}$ (B). (C) AO staining was used to visually represent the inhibitory effect on autophagy by HNK via fluorescence microscopy. The cells were exposed to HNK for 24 h. NSCLC, non-small cell lung cancer cells; HNK, honokiol; AO, acridine orange.

combinations. The results demonstrated that HNK, CQ or 3-MA all induced the expression of p62 in the A549 and H460 cells, but HNK or CQ alone increased the level of LC3-II expression. HNK or CQ alone augmented the level of p62 induced by 3-MA and increased the LC3-II expression level even though 3-MA did not induce the LC3-II expression. However, the combination of HNK and CQ did not clearly change the level of the two autophagic markers as compared to HNK or CQ alone (Fig. 6B). HNK inhibited the fusion of lysosomes and autophagosomes and possessed various characteristics of an autophagy inhibitor such as CQ.

Inhibition of autophagy augments cell death induced by HNK in human NSCLC cells. From the above results, HNK 
A
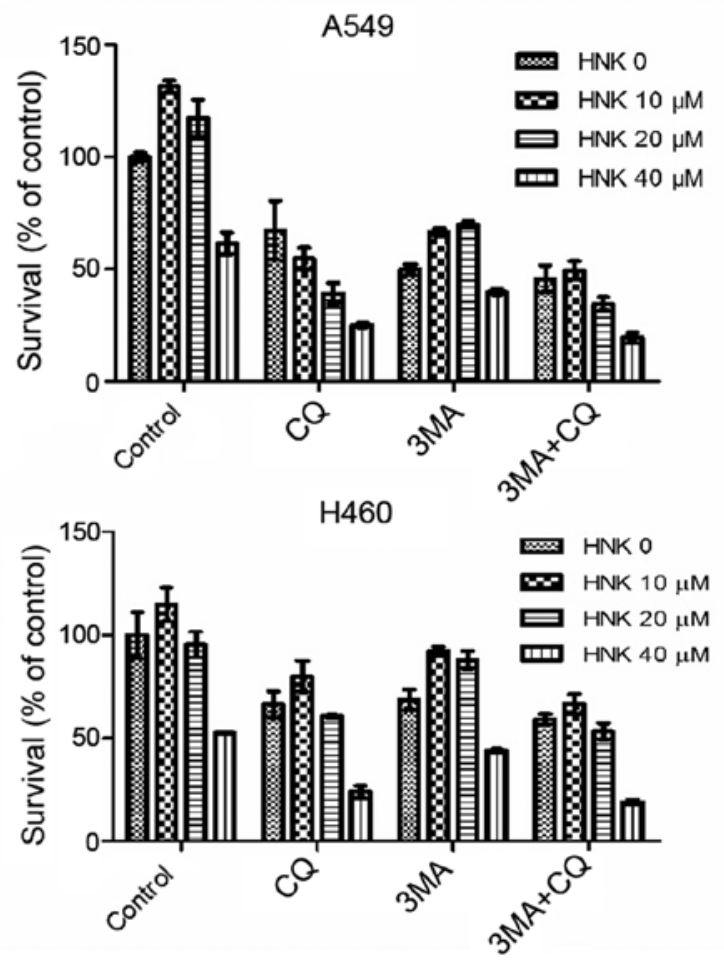

B
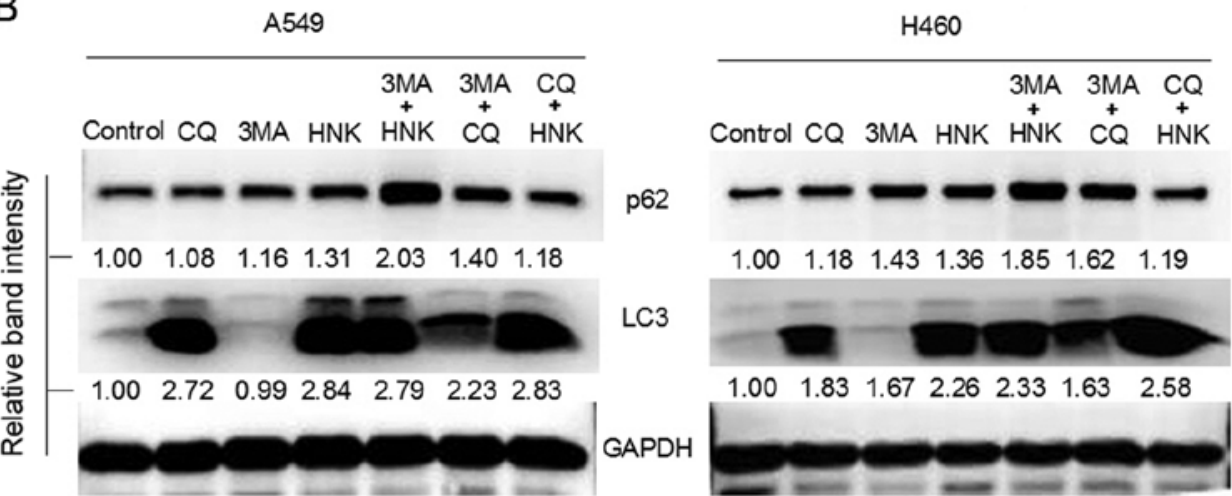

Figure 6. HNK combined with autophagy inhibitors affects the proliferation and autophagy-related protein expression in human NSCLC cells. (A) Cell viability of the A549 and H460 cells in response to CQ, 3-MA and HNK in the indicated groups was investigated by SRB assay. The cells were treated with CQ $(20 \mu \mathrm{M}), 3-\mathrm{MA}(4 \mathrm{mM})$ and HNK $(10,20,40 \mu \mathrm{M})$ groups for $48 \mathrm{~h}$. Each independent experiment was performed in triplicate, $\mathrm{n}=3$. (B) The expression of p62 and LC 3 in the A549 and H460 cells in response to CQ, 3-MA and HNK for $24 \mathrm{~h}$ was assessed by western blotting. The number under each band are the ratio of the optical density of the band relative to that of the untreated group. HNK, honokiol; CQ, chloroquine; SRB, sulforhodamine $\mathrm{B}$.

inhibited autophagy during our experiments. Thus, rapamycin and CQ, which induces and inhibits autophagy respectively, were used to verify the role of autophagy in the cell death of human NSCLC cells induced by HNK. As shown in Fig. 7A, rapamycin did not enhance the anticancer effect of $\mathrm{HNK}$ at the low concentrations of 10 and $20 \mu \mathrm{M}$ or CQ in both cell lines, but an enhanced effect of CQ and HNK was noted. The western blot results (Fig. 7B) showed that rapamycin reduced p62 expression and increased LC3 expression in the A549 and H460 cells when in combination with CQ or HNK. Surprisingly, HNK increased the expression of p62 and LC3 similar to $\mathrm{CQ}$, and there is no obvious increase in the expression of p62 and LC3 when combining HNK and CQ.

$H N K$ induces the expression of cathepsin D. Cathepsin D, a member of the lysosomal aspartyl cathepsin family, plays a crucial role in cell fate and tissue homeostasis through apoptosis (21). Based on the above results, HNK induced apoptosis, thus we investigated the effect of HNK on cathepsin D expression. In the A549 and H460 cells, HNK increased the level of cathepsin D protein in both a time-dependent and concentration-dependent manner. HNK at $20 \mu \mathrm{M}$ increased expression of cathepsin D at $24 \mathrm{~h}$ after treatment in both cell lines (Fig. 8A). Upregulation of cathepsin D was observed even at $4 \mathrm{~h}$ post HNK treatment. The increase in cathepsin D by HNK was maintained for up to $24 \mathrm{~h}$ in the A549 and H460 cells (Fig. 8B).

In order to further determine the role of cathepsin D in the apoptosis-induction of HNK, cathepsin D siRNA and pepstatin A (PepA) as an aspartate protease inhibitor of cathepsin D were used in the experiments. As shown in Fig. 8C, the siRNA efficiently knocked down cathepsin 

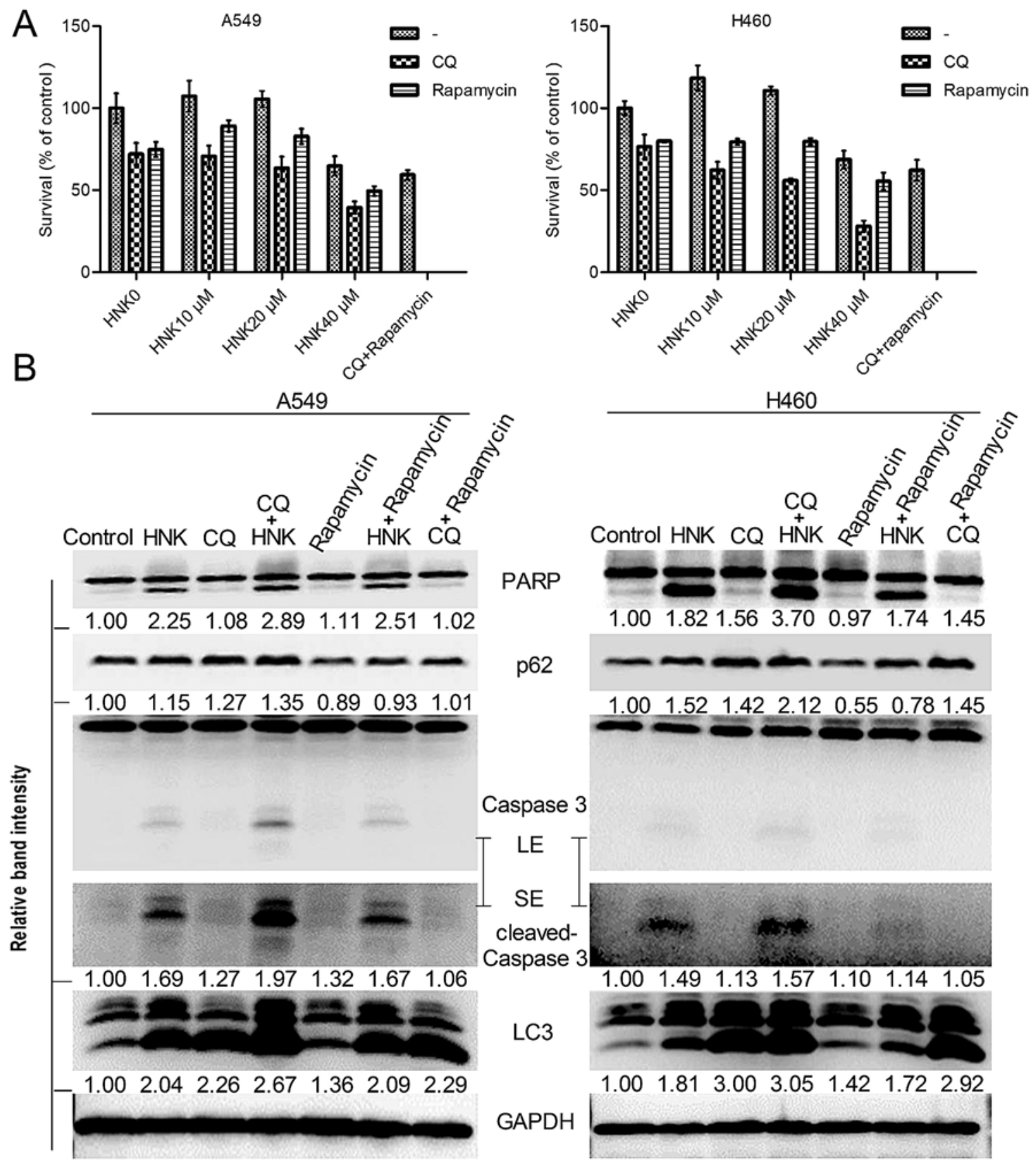

Figure 7. HNK or CQ combined with rapamycin inhibits the proliferation and affects the expression of apoptosis- and autophagy-associated proteins in the A549 and H460 cells. (A) The cells were pre-treated with CQ $(20 \mu \mathrm{M})$ or rapamycin $(400 \mathrm{nM})$ for $1.5 \mathrm{~h}$ and then $\mathrm{HNK}(0,10,20,40 \mu \mathrm{M})$ was added to the culture system. The cell survival ratio was tested via MTT assay. (B) The cells were pre-treated with CQ $(20 \mu \mathrm{M})$ or rapamycin $(400 \mathrm{nM})$ for $1.5 \mathrm{~h}$, and then HNK ( $40 \mu \mathrm{M}$ for the A549 cells and $30 \mu \mathrm{M}$ for the $\mathrm{H} 460$ cells) was added to the culture system. The expression of p62, LC3-I/II, PARP and caspase-3 were assessed with western blotting. The number under each band is the ratio of the optical density of the band relative to that of the untreated group. SE refers to short exposure. LE refers to long exposure. $\mathrm{HNK}$, honokiol; $\mathrm{CQ}$, chloroquine.

D expression in the A549 and H460 cells with or without HNK treatment. Notably, the expression of PARP and cleaved caspase-3 was elevated after the gene of cathepsin D was knocked down in both cell lines treatment with HNK (Fig. 8C). In addition, PepA significantly potentiated the inhibitory effect on the proliferation of the A549 and H460 cells by HNK (Fig. 8D).

\section{Discussion}

Cancer cells are able to adapt themselves to extreme conditions such as oxygen and nutrient-poor stress, which lead to tumor growth, resistance and relapse. Stress conditions induce cancer cells to autophagy and it enables cancer cells to survive by providing biosynthetic precursors, maintaining energy production, and clearing injured cell components (22). Clearly, autophagy plays an important role in tumor cell survival under extreme stress, thus inhibition of autophagy may become a novel therapeutic strategy for cancer treatment $(23,24)$. It has been reported that chemical inhibitors of autophagy induce the death of cancer cells and sensitize the antitumor activity of a broad array of anticancer agents (25). CQ, an autophagic inhibitor, is being used as a chemosensitizer in clinical trials (11). HNK, a small unsymmetrical natural polyphenol, has been 

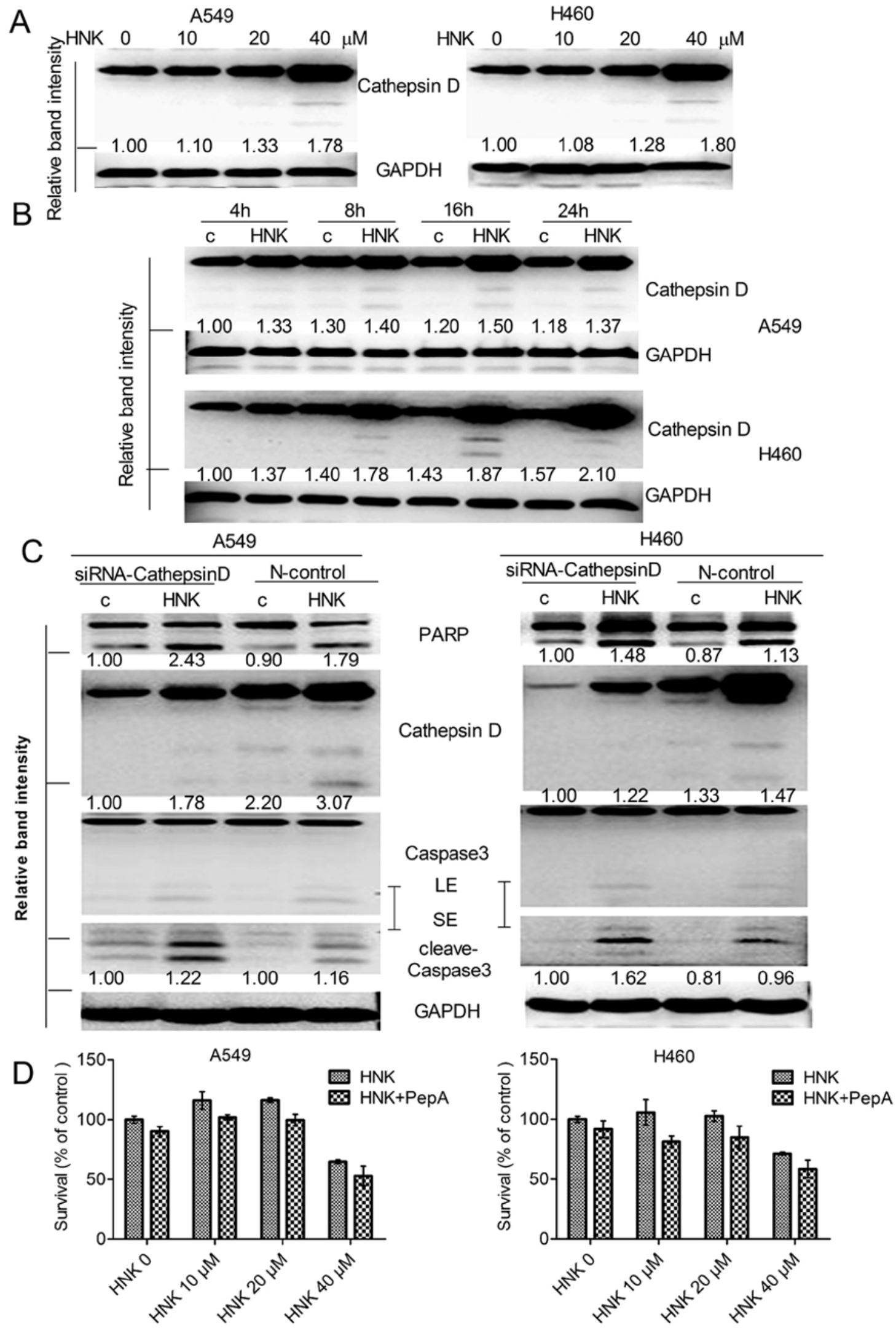

Figure 8. HNK induces apoptosis in a cathepsin D-involved manner. (A and B) The expression of cathepsin D was assessed by western blotting assay after the A549 and $\mathrm{H} 460$ cells were treated with the indicated concentrations of $\mathrm{HNK}$ (A), and with $40 \mu \mathrm{M}$ (A549 cells) and $30 \mu \mathrm{M}$ (H460 cells) HNK respectively, for the indicated times (B). The number under each band indicates the ratio of the optical density of the band relative to that of the untreated group (A) or the control at $4 \mathrm{~h}$ (B). (C) Cathepsin D knockdown increased HNK-induced apoptosis. Cathepsin D RNAi was performed as described in Materials and methods. The A549 and H460 cells were transfected with $\mathrm{N}$-control and cathepsin D siRNA and treated with HNK for another $24 \mathrm{~h}$, and the apoptosis-related proteins were examined by western blotting. The number under each band is the ratio of the optical density of the band relative to that of the untreated group for siRNA group. SE refers to short exposure. LE refers to long exposure. (D) Pep A enhanced the antitumor effect of HNK. The cells were pretreated with pepstatin A for $16 \mathrm{~h}$, and then $\operatorname{HNK}(0,10,20$ and $40 \mu \mathrm{M})$ was added for another $48 \mathrm{~h}$. Induction of apoptosis was investigated via the MTT assay. The number under each blot indicate the intensity of the blot relative to that of the control (the blank group). HNK, honokiol; PepA, pepstatin A. 
used in the clinic as a traditional Chinese drug for many years. Also, autophagy is involved in the pharmacological effects of HNK. In previous studies, antitumor drugs are often combined with CQ. In the present study, the inhibitory effect of HNK or coupled with CQ on autophagy and its crosstalk with apoptosis in NSCLC cells were investigated.

SRB assay, apoptosis analysis, western blotting and animal experiments were used to investigate the enhanced antitumor effect of the combination of HNK and CQ in vitro and in vivo. From the results, we demonstrated a more potent proliferation inhibition of NSCLC cells in the HNK combined with CQ group than that in the group treated with HNK or CQ alone. HNK induced apoptosis of human NSCLC cells and CQ augmented the efficacy of the cell death caused by HNK. A pan-caspase inhibitor (zVAD.fmk) significantly decreased the cleaved caspase- 3 and PARP expression, indicating the existence of caspase-dependent cell death in our research system. The effects on the viability of NSCLC cells in vitro or the growth of the H460 xenografts in vivo by HNK and/ or CQ both confirmed that they produced a marked antitumor effect. Furthermore, the percentage of tumor growth reduction in the HNK combined with CQ group was the highest among the three groups: HNK, CQ, and HNK combined with CQ. Based on the fact that autophagy influences the pharmacological function of HNK and CQ, we detected the expression level of LC3-II in the tumor tissues in all of the experimental groups. The results showed that HNK had the obvious ability to increase the expression of LC3-II protein in vivo, which indicated that autophagy was involved in the antitumor effect of HNK or coupled with CQ in vivo.

In order to illustrate the clear role of autophagy, we examined the expression level of autophagy-related proteins, p62 and LC3-II, in vitro. p62 is identified as the substrate of autophagy degraded by autophagosomes (26). LC3 is the most widely used biomarker of autophagosome formation with the capability of inducing autophagy $(27,28)$. HCQ inhibited autophagy through augumenting p62 and LC3-II proteins (29). In our experiment, we found that HNK increased the expression levels of p62 and LC3-II, showing that HNK inhibited autophagy in the NSCLC cells via a similar mechanism to CQ by disrupting autolysosome function. It is well-known that 3-MA suppresses the start of autophagy, but CQ exerts effects at the late phase of autophagy. 3-MA decreased the accumulation of LC3-II induced by CQ, but not by HNK, which indicated that the inhibitory effect on autophagy by HNK was more potent than that by CQ, in the NSCLC cells. HNK induces autophagy in glioblastoma multiforme and prostate cancer cells. Therefore, we proposed that the influence of autophagy in the antitumor effect of HNK was dependent on the type of cell line.

Autophagy inhibitors or activators were applied to determine the role of autophagy in the tumor growth of NSCLC following treatment with $\mathrm{HNK}$ and combined with CQ. Treatment with combinations of $\mathrm{CQ}+\mathrm{HNK}$ and 3-MA+HNK indicated that there were enhanced effects on the viability of the NSCLC cells or the growth of the H460 xenografts in vivo by CQ combined with HNK. HNK and CQ play a role in the late phase of autophagy via a similar mechanism. Thus the combination of drugs with a similar mechanism enhanced the antitumor effects. Furthermore, we compared the inhibitory effect on the proliferation of the combinations of rapamycin+CQ and $\mathrm{HNK}$, and CQ+HNK in the H460 and A549 cells. Rapamycin did not augment the anticancer effect of HNK at the low concentrations of 10 and $20 \mu \mathrm{M}$ or CQ in both cell lines, but an enhanced effect of CQ and HNK was noted. The results showed that the inhibition of autophagy by HNK decreased the growth of NSCLC cells and further proved that HNK suppressed autophagy at the late phase similar to CQ. The inhibitory effect of autophagy was involved in the antitumor effect of HNK coupled with CQ.

Cathepsin D, an aspartic protease, is localized in lysosomes under normal conditions and participates in many physiological processes, playing a critical role in protein degradation, modulation of cell death, and epithelial differentiation. However, cathepsin D is overexpressed and secreted aberrantly in different types of cancers including prostate cancer and lung adenocarcinoma $(30,31)$, and its level is correlated with growth, invasion, metastasis and angiogenesis of cancer. It is reported that overexpression of wild-type cathepsin D clearly reduces the apoptosis of cancer cells (32). The present study demonstrated that HNK decreased the proliferation of NSCLC cells and induced apoptosis. Thus the role of cathepsin D was investigated in our research. In fact, HNK increased the cathepsin D expression level in a time-dependent and dose-dependent manner and cathepsin D siRNA potentiated the apoptosis mediated by HNK. PepA, a cathepsin D inhibitor, enhanced the inhibitory effect on the proliferation of NSCLC cells by HNK. Thus, we postulated that cathepsin D exerted a protective effect on the apoptosis induced by HNK. Cathepsin D partly participated in the antitumor effect of $\mathrm{HNK}$ or in combination with CQ.

In summary, our results showed enhanced effects of the combination of HNK and CQ on the inhibition of proliferation in vitro and the reduction in growth in vivo in NSCLC. We also demonstrated that HNK increased the expression levels of p62 and LC3-II protein and inhibited autophagy similar to CQ via a similar mechanism. Inhibition of autophagy and induction of apoptosis are involved in the antitumor effect of HNK or in combination with CQ in a cathepsin D-mediated and caspase-dependent manner. HNK disturbs autophagy in vitro and in vivo, and exhibits a potent cytotoxic effect and may be a novel antitumor sensitizer similar to CQ.

\section{Acknowledgements}

The present study was supported by grants from the Natural Science Foundation of China (no. 81373437) and the 'Significant New Drug Development' of the Science and Technology Major Projects of China (no. 2012ZX09301002-001-022-01).

\section{References}

1. Arora S, Singh S, Piazza GA, Contreras CM, Panyam J and Singh AP: Honokiol: A novel natural agent for cancer prevention and therapy. Curr Mol Med 12: 1244-1252, 2012.

2. Banerjee P, Basu A, Arbiser JL and Pal S: The natural product honokiol inhibits calcineurin inhibitor-induced and Ras-mediated tumor promoting pathways. Cancer Lett 338: 292-299, 2013.

3. Lai YJ, Lin CI, Wang CL and Chao JI: Expression of survivin and p53 modulates honokiol-induced apoptosis in colorectal cancer cells. J Cell Biochem 115: 1888-1899, 2014. 
4. Raja SM, Chen S, Yue P, Acker TM, Lefkove B, Arbiser JL, Khuri FR and Sun SY: The natural product honokiol preferentially inhibits cellular FLICE-inhibitory protein and augments death receptor-induced apoptosis. Mol Cancer Ther 7: 2212-2223, 2008.

5. Chen YJ, Wu CL, Liu JF, Fong YC, Hsu SF, Li TM, Su YC, Liu SH and Tang CH: Honokiol induces cell apoptosis in human chondrosarcoma cells through mitochondrial dysfunction and endoplasmic reticulum stress. Cancer Lett 291: 20-30, 2010.

6. Chang KH, Yan MD, Yao CJ, Lin PC and Lai GM: Honokiol-induced apoptosis and autophagy in glioblastoma multiforme cells. Oncol Lett 6: 1435-1438, 2013.

7. Hahm ER, Sakao K and Singh SV: Honokiol activates reactive oxygen species-mediated cytoprotective autophagy in human prostate cancer cells. Prostate 74: 1209-1221, 2014.

8. Bu Q, Liu X, Zhu Y, Liu Y and Wang Y: w007B protects brain against ischemia-reperfusion injury in rats through inhibiting inflammation, apoptosis and autophagy. Brain Res 1558: 100-108, 2014.

9. Lao Y, Wan G, Liu Z, Wang X, Ruan P, Xu W, Xu D, Xie W, Zhang $\mathrm{Y}, \mathrm{Xu} \mathrm{H}$, et al: The natural compound oblongifolin $\mathrm{C}$ inhibits autophagic flux and enhances antitumor efficacy of nutrient deprivation. Autophagy 10: 736-749, 2014.

10. Gros F and Muller S: Pharmacological regulators of autophagy and their link with modulators of lupus disease. $\mathrm{Br}$ Pharmacol 171: 4337-4359, 2014.

11. Nagelkerke A, Bussink J, Geurts-Moespot A, Sweep FC and Span PN: Therapeutic targeting of autophagy in cancer. Part II: Pharmacological modulation of treatment-induced autophagy. Semin Cancer Biol 31: 99-105, 2014.

12. Poklepovic A and Gewirtz DA: Outcome of early clinical trials of the combination of hydroxychloroquine with chemotherapy in cancer. Autophagy 10: 1478-1480, 2014.

13. Vogl DT, Stadtmauer EA, Tan KS, Heitjan DF, Davis LE, Pontiggia L, Rangwala R, Piao S, Chang YC, Scott EC, et al: Combined autophagy and proteasome inhibition: A phase 1 trial of hydroxychloroquine and bortezomib in patients with relapsed/refractory myeloma. Autophagy 10: 1380-1390, 2014.

14. Viola G, Bortolozzi R, Hamel E, Moro S, Brun P, Castagliuolo I, Ferlin MG and Basso G: MG-2477, a new tubulin inhibitor, induces autophagy through inhibition of the Akt/mTOR pathway and delayed apoptosis in A549 cells. Biochem Pharmacol 83: 16-26, 2012.

15. Wang D, Wang Z, Tian B, Li X, Li S and Tian Y: Two hour exposure to sodium butyrate sensitizes bladder cancer to anticancer drugs. Int J Urol 15: 435-441, 2008.

16. Liu F, Shang Y and Chen SZ: Chloroquine potentiates the anti-cancer effect of lidamycin on non-small cell lung cancer cells in vitro. Acta Pharmacol Sin 35: 645-652, 2014.

17. Wang X, Beitler JJ, Wang H, Lee MJ, Huang W, Koenig L, Nannapaneni S, Amin AR, Bonner M, Shin HJ, et al: Honokiol enhances paclitaxel efficacy in multi-drug resistant human cancer model through the induction of apoptosis. PLoS One 9: e86369, 2014.
18. Hu W, Chen SS, Zhang JL, Lou XE and Zhou HJ: Dihydroartemisinin induces autophagy by suppressing NF- $\mathrm{KB}$ activation. Cancer Lett 343: 239-248, 2014.

19. Hasson SA, Kane LA, Yamano K, Huang CH, Sliter DA, Buehler E, Wang C, Heman-Ackah SM, Hessa T, Guha R, et al: High-content genome-wide RNAi screens identify regulators of parkin upstream of mitophagy. Nature 504: 291-295, 2013.

20. Zhang J, Chiu J, Zhang H, Qi T, Tang Q, Ma K, Lu H and Li G: Autophagic cell death induced by resveratrol depends on the $\mathrm{Ca}(2+) / \mathrm{AMPK} / \mathrm{mTOR}$ pathway in A549 cells. Biochem Pharmacol 86: 317-328, 2013.

21. Marques C, Oliveira CS, Alves S, Chaves SR, Coutinho OP, Côrte-Real M and Preto A: Acetate-induced apoptosis in colorectal carcinoma cells involves lysosomal membrane permeabilization and cathepsin D release. Cell Death Dis 4: e507, 2013

22. Esteve JM and Knecht E: Mechanisms of autophagy and apoptosis: Recent developments in breast cancer cells. World J Biol Chem 2: 232-238, 2011.

23. Bincoletto C, Bechara A, Pereira GJ, Santos CP, Antunes F, Peixoto da-Silva J, Muler M, Gigli RD, Monteforte PT, Hirata H, et al: Interplay between apoptosis and autophagy, a challenging puzzle: New perspectives on antitumor chemotherapies. Chem Biol Interact 206: 279-288, 2013.

24. Leone RD and Amaravadi RK: Autophagy: A targetable linchpin of cancer cell metabolism. Trends Endocrinol Metab 24: 209-217, 2013.

25. Sharma N, Thomas S, Golden EB, Hofman FM, Chen TC, Petasis NA, Schönthal AH and Louie SG: Inhibition of autophagy and induction of breast cancer cell death by mefloquine, an antimalarial agent. Cancer Lett 326: 143-154, 2012.

26. Moscat J and Diaz-Meco MT: p62: a versatile multitasker takes on cancer. Trends Biochem Sci 37: 230-236, 2012.

27. Nakatogawa H, Ichimura Y and Ohsumi Y: Atg8, a ubiquitin-like protein required for autophagosome formation, mediates membrane tethering and hemifusion. Cell 130: 165-178, 2007.

28. Xie Z, Nair U and Klionsky DJ: Atg8 controls phagophore expansion during autophagosome formation. Mol Biol Cell 19: 3290-3298, 2008

29. Pruitt FL, He Y, Franco OE, Jiang M, Cates JM and Hayward SW: Cathepsin D acts as an essential mediator to promote malignancy of benign prostatic epithelium. Prostate 73: 476-488, 2013.

30. Saleem A, Dvorzhinski D, Santanam U, Mathew R, Bray K, Stein M, White E and DiPaola RS: Effect of dual inhibition of apoptosis and autophagy in prostate cancer. Prostate 72: 1374-1381, 2012.

31. Mimae T, Tsuta K, Maeshima AM, Okada M, Asamura H, Kondo $\mathrm{T}$ and Tsuda H: Cathepsin D as a potential prognostic marker for lung adenocarcinoma. Pathol Res Pract 208: 534-540, 2012.

32. Hah YS, Noh HS, Ha JH, Ahn JS, Hahm JR, Cho HY and Kim DR: Cathepsin D inhibits oxidative stress-induced cell death via activation of autophagy in cancer cells. Cancer Lett 323: 208-214, 2012 